\title{
Oscillatory secular modes: the thermal micropulses
}

\author{
A. Gautschy ${ }^{1}$ and L. G. Althaus ${ }^{2}$ \\ 1 Wetterchrüzstr. 8c, 4410 Liestal, Switzerland \\ e-mail: alfred@gautschy.ch \\ 2 Facultad de Ciencias Astronómicas y Geofísicas, Universidad Nacional de La Plata, Paseo del Bosque S/N (1900) La Plata and \\ Instituto de Astrofisica La Plata (IALP), Argentina
}

Received 31 May 2006 / Accepted 6 June 2007

\begin{abstract}
Aims. Stars in the narrow mass range of about 2.5 and $3.5 M_{\odot}$ can develop a thermally unstable He-burning shell during its ignition phase. We study, from the point of view secular stability theory, these so-called thermal micropulses and investigate their properties; the thermal pulses constitute a convenient conceptual laboratory to look thoroughly into the physical properties of a helium-burning shell during the whole thermally pulsing episode.

Methods. Linear stability analyses were performed on a large number of $3 M_{\odot}$ star models toward the end of their core helium-burning and the beginning of the double shell burning phase. The stellar models were not assumed to be in thermal equilibrium.

Results. The thermal mircopulses - and we conjecture all other thermal pulse episodes encountered by shell burning stars - can be understood as the nonlinear finite-amplitude realization of an oscillatory secular instability that prevails during the whole thermal pulsing episode. Hence, the cyclic nature of the thermal pulses can be traced back to a linear instability concept.
\end{abstract}

Key words. stars: AGB and post-AGB - stars: interiors - stars: oscillations

\section{Introduction}

According to the published literature, Gurevich \& Lebedinski (1947) seem to have been the first to study the thermal stability of nuclear-burning shells in stars; the authors aimed to reveal the physical nature of novae and supernovae. The work of Gurevich \& Lebedinksi apparently went unnoticed outside of the Soviet Union and 15 years passed before the field advanced: The seminal paper of Schwarzschild \& Härm (1965) reported the thermal instability of the He-burning shell in a $1 M_{\odot}$ asymptotic giantbranch (AGB) star. The instability was stumbled upon once a sufficiently small time-step of the evolution computation was chosen during the pertinent evolutionary phase. Independently of Schwarzschild \& Härm (1965), Weigert (1965, 1966) observed the same phenomenon at the beginning of the doubleshell burning phase of a $5 M_{\odot}$ star model. Weigert's computations already indicated the cyclic nature of the instability. Rose (1966), studying the advanced evolution of a $0.53 M_{\odot}$ helium star, also picked up thermal pulses once the evolutionary timestep became sufficiently short. As Rose was interested in the evolution to the white-dwarf domain of He-star models, he chose large time-steps across the helium shell burning phase so that he captured only the last three thermal pulse cycles of the instability episode. In any case, Rose (1966) emphasized, as did Weigert, the cyclic nature of the phenomenon and he was the first to present eigenfunctions in the surroundings of the unstable nuclear burning shell.

Already Ledoux (1962) noted that, in principle at least, the secular stability problem admits of complex eigensolutions. The stability analysis of thin nuclear-burning shells was extended in Härm \& Schwarzschild (1972) wherein the thermal pulses, eventually considered as a cyclic phenomenon, were associated with the star's secular modes. The authors connected the onset of the thermal pulses with an overstable oscillatory secular mode.
After the first few pulse cycles, however, "[...] the oscillatory instability gives way [...] to a simple exponential instability which causes the repetitive helium-shell flashes described in earlier investigations [...]" as stated by Härm \& Schwarzschild (1972). This last statement seems to have been taken as the final word on the issue and was perpetuated as such in the astronomical literature thereafter.

Despite the apparent settlement of the case, the point of view adopted by Härm \& Schwarzschild (1972) continued to intrigue as it does not explain the cause of the cyclic nature of the thermal pulses once the oscillatory instability gives way to an exponential one. The numerical studies showed that the period of the initial oscillatory secular mode was close to the length of the developing thermal pulse cycles of the helium shell. Why or how the star remembered the linear result so closely remained unexplained (Hansen 1978). If the cyclic nature of the thermal pulses is indeed a nonlinear phenomenon that escapes accessibility of a linear analysis, then the "relaxation oscillations" of the thermalpulse phase would be a realization of hard self-excited oscillations (Cox 1980) in stars (see also Buchler \& Perdang 1979).

Besides the thermal pulses that are associated with secularly unstable thin helium-burning shells in low- and intermediatemass stars along the asymptotic giant branch, secularly unstable shell-burning is also encountered at the onset of off-center helium-burning in low-mass stars as they evolve from the tip of the red-giant branch onto the horizontal branch (e.g. Thomas 1967; Despain 1981). But also more exotic episodes of stars' lives can get entangled in He-shell instabilities: X-ray bursts as observed in mass-accreting neutron stars (e.g. Hansen \& Van Horn 1975; Bildsten 1995). Even hydrogen-burning shells on accreting white dwarfs can become cyclically unstable (e.g. Giannone \& Weigert 1967; Cassisi et al. 1998, and references in the latter) via the same physical mechanism. Hence, a solid 
understanding of the thermal pulses is beneficial for disparate fields of stellar astrophysics.

This paper deals with those thermal pulses that are encountered when, under suitable circumstances, a helium shell builds up and ignites at the end of core He burning of a $3 M_{\odot}$ star (Mazzitelli \& D'Antona 1986). Applying the simple, local instability criterion for thin nuclear-burning shells of Schwarzschild \& Härm (1965), Mazzitelli \& D’Antona (1986) concluded that the instability was physical and due to the same mechanism as encountered later, higher up along the AGB. The thermal pulses at the onset of He shell burning were termed thermal micropulses (ThMPs) as the surface luminosity varies only marginally as compared to the variation during a thermal pulse on the advanced AGB. In an attempt to evolve a star from the main sequence to the terminal Debye-cooling stage as a white dwarf, thermal micropulses were also encountered by Althaus et al. (2002) in stellar models for which semiconvection was neglected in the computations. It seems that only stars in the narrow mass range of 2.5-3.5 $M_{\odot}$ develop ThMPs under suitable conditions.

The evolutionary tracks of stars undergoing ThMPs (cf. Mazzitelli \& D'Antona 1986; Althaus et al. 2002) show that the manifestation of the pulses in the stars' observables are minimal. The pulse amplitudes in the total luminosity are small and the time-scale of the pulses so short that effects on a population of stars on the early second ascent of the giant branch are very unlikely to be detectable even in large stellar aggregates. Despite ThMPs being unlikely to be observable or possibly even realized in nature, their very existence in star models constitutes a welcome opportunity: with ThMPs, we can theoretically study the stability properties of a secularly unstable nuclear-burning shell from onset of the instability to the very end when the shell stabilizes itself again. In particular, the self-stabilization of the shell distinguishes the ThMPs from thermal pulses along the upper AGB. Furthermore, we expect that the properties of the secular mode spectrum of the ThMP phase are also representative for the thermally pulsing stars on the advanced AGB.

In the following, we use the ThMP phase as an idealized laboratory to study the episode of cyclic thermal pulses once again from the point of view of linear secular stability theory. We take advantage of the result of Gabriel (1972) that over timescales that are short compared with a star's nuclear time-scale its quasi-static evolution can be expanded in terms of secular eigendata. We follow the pertinent part of the secular eigenspectrum throughout the whole ThMP episode and we show that ThMPs can be understood as an oscillatory secular instability; hence nonlinear theory via relaxation oscillations does not have to be invoked to explain the existence of the cyclic instability. Nonlinear effects, on the other hand, determine the temporal shape of the observables of the pulses and they seem to govern the amplitude evolution of the pulse cycles.

\section{Modeling procedure}

Stellar evolution was computed with the LPCODE code described in Althaus et al. (2002) and Althaus et al. (2003). Convective overshooting was modeled as an exponentially decaying diffusive process above and below every convective shell, including the boundary of the convective core (during the main-sequence and central helium-burning phase) and the convective envelope (for details see Althaus et al. 2003). The nuclear network considered for the stellar modeling accounts explicitly for 16 chemical elements and 34 thermonuclear reaction rates to follow hydrogen and helium burning. Abundance changes of the nuclear species were included by means of a time-dependent scheme that simultaneously treats nuclear evolution and mixing processes. The efficiency of convective mixing was described by appropriate diffusion coefficients which are specified by the treatment of convection. The equation of state included partial ionization, ionic contributions, partially degenerate electrons, and Coulomb interactions. Radiative opacities, including carbon- and oxygen-rich compositions, were those from the OPAL sets (Iglesias \& Rogers 1996), complemented, at low temperatures, with the molecular opacities from Alexander \& Ferguson (1994).

The computations to investigate the linear secular stability properties of the star models were performed with the Riccati code for radial nonadiabatic stellar pulsations; the current version of the code is based on that described in Gautschy \& Glatzel (1990). In addition to the canonical radial pulsation equations, we also used modified stability equations wherein the condition of thermal equilibrium of the background model was relaxed. For completeness, the relevant linear stability equations are presented in Appendix A.

\section{Results}

This section contains the results obtained from the computation of the evolution of a $3 M_{\odot}$ model with initially homogeneously distributed abundances of $X=0.705, Y=0.275$. We followed the star's evolution from the main sequence to stable doubleshell burning on the lower AGB. The second part of this section is devoted to the secular stability analyses performed on the evolutionary star models between approximately the ignition of the helium-burning shell and the termination of the thermal pulses.

\subsection{The micropulses as seen in stellar evolution computations}

Figure 1 shows, on three zoom levels, the evolution on the Hertzsprung-Russell (HR) plane of the $3 M_{\odot}$ star mentioned before. The displayed track resulted from the computations without convective overshooting - thermal micropulses developed only in such stellar models; we will return to this aspect later. Panel (a) captures the star's locus between core hydrogen burning on the main sequence and the establishing of two burning shells at the bottom of the AGB. Core helium burning sets in at the tip of the first giant branch at the upper right of panel (a). Panel (b) shows the stump of a blue loop that develops during core helium burning. After passing through a local luminosity minimum at $\log L / L_{\odot}=1.792$, with a central helium abundance $Y_{\mathrm{c}}=0.8503$, the star's luminosity rises again to pass through a second local luminosity maximum $\left(\log L / L_{\odot}=2.015\right)$. Label A denotes the epoch of the evolution at which the secular stability analyses were started. For ease of presentation, the secular stability results will be displayed mostly as a function of model number; label A corresponds to model number 1300 with $Y_{\mathrm{c}}=0.288$. The helium shell ignited at $Y_{\mathrm{c}}=0.04$; the corresponding model number, 1400 , is labeled along the track in panel (b). At the second local luminosity maximum (model number 1490 in panel c), $Y_{\mathrm{c}}$ dropped to 0.0325 and the triple$\alpha$ energy-generation rate of the nuclear-burning shell reached the same strength as that of core helium burning. Hence, after model 1490, the star's helium burning is shell dominated. Finally, panel (c) zooms in to the cyclically varying luminosity and effective temperature that develop shortly after model 1490. The star passes through a local luminosity minimum after the 6 th pulse cycle. Around this local luminosity minimum, the surface-luminosity variation reaches its maximum with a relative 

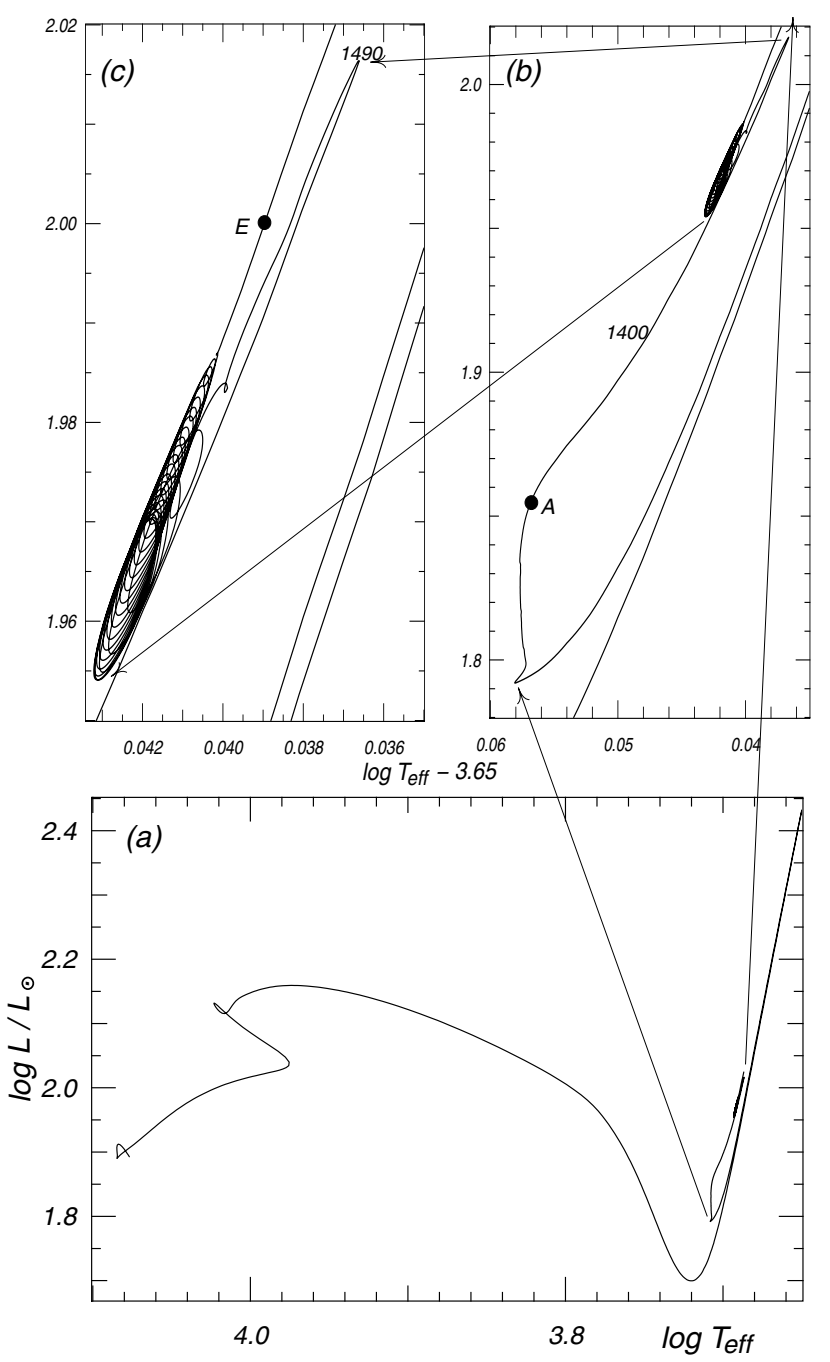

Fig. 1. Evolutionary track of a $3 M_{\odot}$ star with pertinent zoom-ins to the epochs of core He burning (panel b)) and to the development of doubleshell nuclear burning (panel c)). Core He burning starts at the tip of the first giant branch that is seen in the upper right of panel a). The transition from He burning in the core to He burning in a shell leads to a temporary luminosity drop (after model 1490 which is designated in panel c)); during this luminosity decrease, the thermal micropulses set in. After the He-burning shell is well developed, the micropulses die out and the star continues its second ascend of the giant branch towards the asymptotic giant branch. The epochs of the beginning and the end of the linear secular stability analyses are indicated by the letters $\mathrm{A}$ and $\mathrm{E}$, respectively. The location of model numbers 1400 and 1490 along the evolutionary track are given for later reference in the text.

amplitude of 5\%. During the ensuing upward evolution along the lower AGB, the amplitude of the ThMPs decreases continuously (see also Fig. 2). The variability disappears completely at about the epoch labeled by E (model number 3234) when the helium in the center has essentially burned away, i.e. having reached $Y_{\mathrm{c}}=1.15 \times 10^{-3}$.

The temporal evolution of pertinent luminosity components is shown in Fig. 2. At model 1490, the epoch of the onset of the ThMPs, the star has reached an age of about $395 \mathrm{My}$. The amplitude of the luminosity variation in the He-burning shell $\left(L_{\mathrm{He}}\right)$ grows rapidly to reach maximum amplitude - varying between 10 and $110 L_{\odot}-$ in cycle 7 . Since the layers in and above the He shell expand during the increase of the shell's energy production, the hydrogen shell effectively cools so that

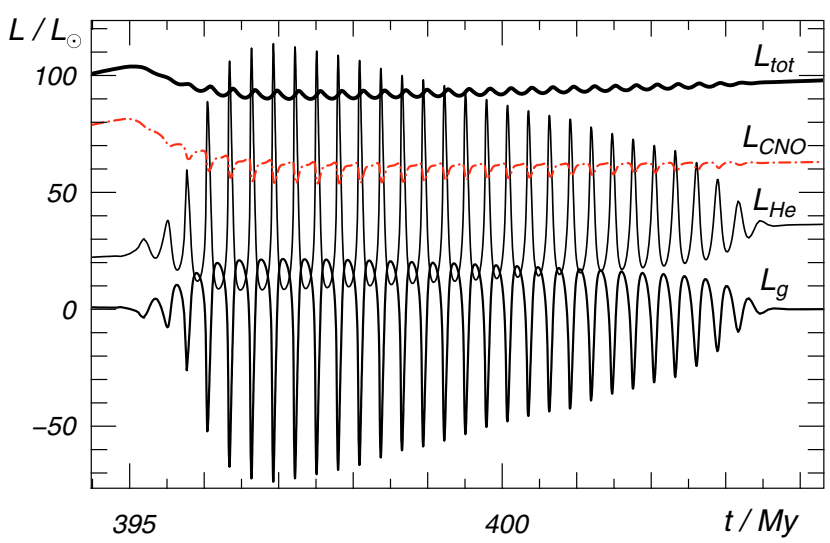

Fig. 2. Variation of various luminosities during the thermal micropulse phase of the evolution of a $3 M_{\odot}$ model. $L_{\text {tot }}$ stands for the total stellar luminosity; it is composed of the helium-burning luminosity $\left(L_{\mathrm{He}}\right)$, the luminosity generated in the hydrogen shell $\left(L_{\mathrm{CNO}}\right)$, and the luminosity induced by expansion/contraction $\left(L_{\mathrm{g}}\right)$ in the own gravitational field. The abscissa measures the age of the star in $10^{6}$ years (My).

its luminosity, $L_{\mathrm{CNO}}$, drops. Figure 2 shows also the mirrorimage behavior of the mechanical work, $L_{\mathrm{g}}$, in the star's gravitational field. Energy is required for expansion during the $L_{\mathrm{He}}$ rise and it is gained during the shrinking of the envelope around $L_{\mathrm{He}}$ minimum. In the sum of the partial luminosities, $L_{\mathrm{tot}} \equiv$ $L_{\mathrm{He}}+L_{\mathrm{CNO}}+L_{\mathrm{g}}$, the total luminosity variation remained always very small, less than $5 \%$. It is mainly $L_{\mathrm{g}}$ which counteracts the strong variation of $L_{\mathrm{He}}$, despite the fact that hydrogen burning mostly dominates the energy production.

Figure 3 shows, for the H-burning shell (top panel) and the He-burning shell (bottom panel), the temporal evolution of physical quantities at the center of the corresponding nuclear-burning shell over the first nine ThMPs. The data for temperature, $T$, density, $\rho$, and the fractional mass, $q$, were shifted vertically to fit into a decently scaled single diagram. The vertical dashed lines in Fig. 3 mark the epochs of maximum $L_{\mathrm{He}}$. The mean radii of the $\mathrm{H}$ - and He-shell slowly increase with time reflecting the slow ascent of the stellar model along the second giant branch (Fig. 1). Notice the quick saturation of the amplitude growth of the radius variation of the He shell after about the first four pulse cycles. The increase in $q$, the fractional mass, of both shells is small. In the He-shell, the density minimum occurs at minimum radius of the shell and vice versa; maximum temperature is well in phase with $L_{\mathrm{He}}$ reflecting the dominating dependence on temperature of the nuclear energy-generation rate of helium burning. The H-shell behaves differently: minimum density agrees with minimum radius and with maximum temperature (this coincides with the maximum-density phase of the He-shell). Hence, minimum radii of $\mathrm{H}$ - and $\mathrm{He}$-shell coincide whereas maximum radius of the $\mathrm{H}$-shell is reached slightly later than maximum radius of the He-shell. The wiggling in $\log \rho$ at the center of the $\mathrm{H}$-shell is an artifact contracted from our definition of the shell center as the gridpoint with maximum nuclear energy generation rate; this maximum slightly shifts in mass and as the density contrast across the H-shell is significant, numerical noise is easily picked up.

After a total of 30 pulse cycles, at about 403.7 My (model number 3234), the ThMPs die out. Hence, the ThMP episode lasts for about $8.7 \mathrm{My}$. The mean pulse-cycle length $\left(\tau_{\mathrm{ThMP}}\right)$ is $0.29 \mathrm{My}$, a value that remains very stable throughout the whole instability episode. 


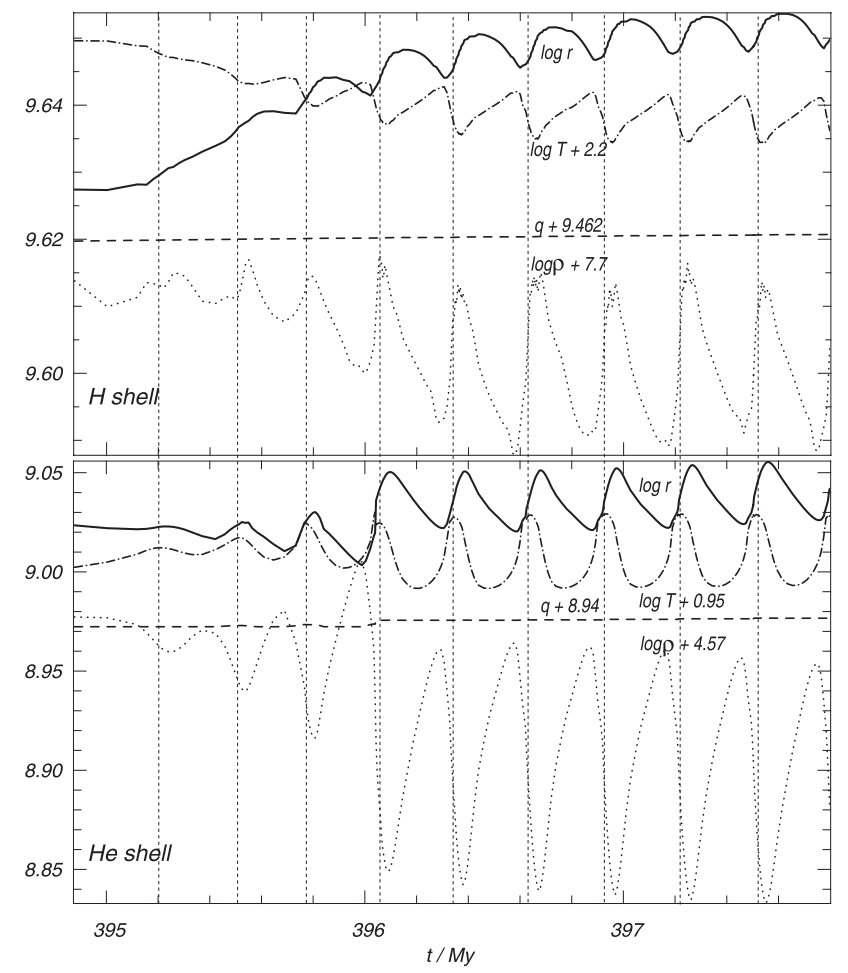

Fig. 3. Temporal variation of selected physical quantities at the center of the hydrogen-burning shell (top panel) and the helium-burning shell (bottom panel). The vertical dashed lines indicate the epochs of the first nine $L_{\mathrm{He}}$ peaks of the ThMP episode. The loci of temperature $(T)$, fractional mass $(q)$, and density $(\rho)$ were shifted vertically to fit the figures that were set up to display the variation of the radii $(r)$ of the shell centers.

\subsection{The secular stability analyses}

Between model numbers 1300 and 3234, i.e. between labels A and E of Fig. 1, we performed linear stability computations on the evolutionary models to study their secular eigenspectra. The physical quantities were assumed to change proportional to $\exp (i \omega t)$. The quantity $\sigma$ that is used in the following is the frequency $\omega$ divided by $\sqrt{3 G M_{*} / R_{*}^{3}}$ with $G$ being the gravitational constant. To obtain approximate e-folding times in mega-years in Fig. 4, compute $5.12 \times 10^{-9} / \sigma_{\mathrm{I}}$.

For the stability computations, we relaxed the requirement of thermal equilibrium of the stellar models; the assumption of complete equilibrium is what is usually implemented in stellar pulsation analyses. For the secular problem, in particular when pulses develop, $L_{\mathrm{g}}$ contributions might be significant so that refraining from the assumption of thermal equilibrium appears more appropriate. The linear stability equations, containing approximations for the thermal imbalance terms, are given in Appendix A.

The left panel of Fig. 4 shows $\sigma_{\text {I }}$, the imaginary parts, of the lowest few secular eigenmodes as a function of model number. Complex conjugate eigensolutions (oscillatory secular modes) are indicated by full dots. Dot-free lines indicate vanishing realparts of $\sigma$, i.e. purely monotonic modes. For better correlation of the linear results with the evolutionary computations, the righthand panel of Fig. 4 plots the $L_{\mathrm{He}}$ oscillations. For completeness, the age in mega-years is labeled along the rightmost ordinate of the figure.

The complexity of the secular eigenspectrum is considerable; and this is so already before the onset of the ThMPs.
During the late core helium-burning phase, the modal diagram is a complicated web of complex-conjugate modes unfolding into pairs of non-oscillatory modes that later merge again into complex-conjugate solutions in different arrangements. In all circumstances, the number of modes involved is conserved.

At model 1300, two unstable monotonic secular modes were encountered. As the core dominance of the nuclear burning ceased, the strength of the instability diminished. Eventually, the most unstable secular modes merged with other branches into oscillatory modes. Most of them became or remained stable during the early helium shell-burning phase. A discussion of the secular-stability behavior during the core He-burning phase of intermediate-mass stars will be re-addressed elsewhere.

The onset of the ThMP phase manifested itself in the least damped oscillatory secular mode going overstable. The first two ThMP cycles appeared as unstable oscillatory secular modes in the linear analysis. Beginning with cycle three, the unstable complex conjugate eigensolution unfolded into a pair of monotonic modes during the maximum of $L_{\mathrm{He}}$ within a cycle. During phases of reduced efficiency of the helium burning shell, the two monotonic modes merged to enter the next cycle through a short damped episode. The cyclic unfolding of the unstable oscillatory secular mode continued to cycle 21 after which the amplitude of the ThMP was seemingly small enough for the unstable secular mode to remain purely oscillatory. The ThMPs, as seen in $L_{\mathrm{He}}$ (e.g. right panel of Fig. 4), terminate by the dominant unstable oscillatory secular mode going stable but remaining oscillatory.

The details of the behavior of the complex eigenvalue $\sigma$ throughout the phases of the ThMP episode must not be overinterpreted. After all, the stability analyses were performed on the thermally pulsing models themselves ${ }^{1}$. Nevertheless, to gain access to the conditions under which complex secular eigenvalues unfold into real pairs, secular analyses on the full cycles of ThMP models might prove helpful.

In addition to the unstable, mainly oscillatory secular mode, Fig. 4 shows a damped oscillatory mode, $\mathrm{O}_{2}$ that never unfolded during the whole ThMP episode. The oscillatory secular mode baptized $\mathrm{O}_{3}$, on the other hand, unfolded again cyclically into pairs of monotonic modes, but in this case during the minimum phases of $L_{\mathrm{He}}$; Fig. 4 shows only one of the monotonic branches of $\mathrm{O}_{3}$ since a follow-up of both turned out to be computationally cumbersome due to other close-lying secular modes.

In the range of the $\sigma_{\mathrm{I}}$ domain considered here, we encountered only three oscillatory or partially oscillatory secular modes. At least in the $\sigma_{\mathrm{I}}-$ model number (or age) diagram these oscillatory modes overlap with purely monotonic secular modes. Except for the mode with the longest time-scale, all of the monotonic modes are always damped. Note that the crossing of modes in Fig. 4 is a projection effect due to plotting $\sigma_{\text {I }}$ only instead of the complex $\sigma$; on the complex $\sigma$ plane, the modes are well separated.

The secular mode with the smallest $\sigma_{\mathrm{I}}$ values and which remains always monotonic switches between weakly stable and

\footnotetext{
1 In analogy with the pulsation theory of stars, our linear secular analyses can be compared to performing linear pulsation analyses on nonlinearly pulsating star models. The period of pulsation derived from such a linear analysis varies cyclically proportional to $R_{*}(t)^{3 / 2}$ where $R_{*}(t)$ is the nonlinearly pulsating stellar radius. Also the damping/excitation rate (our $\sigma_{\mathrm{I}}$ here) varies along a pulsation cycle. In particular when the nonlinearly pulsating model is hottest, the pulsational excitation by the $x$-mechanism of the stellar envelope is diminished as the driving zone lies too close to the surface; at the phase of lowest surface temperature, excitation is diminished as the driving region lies too deep in the envelope.
} 


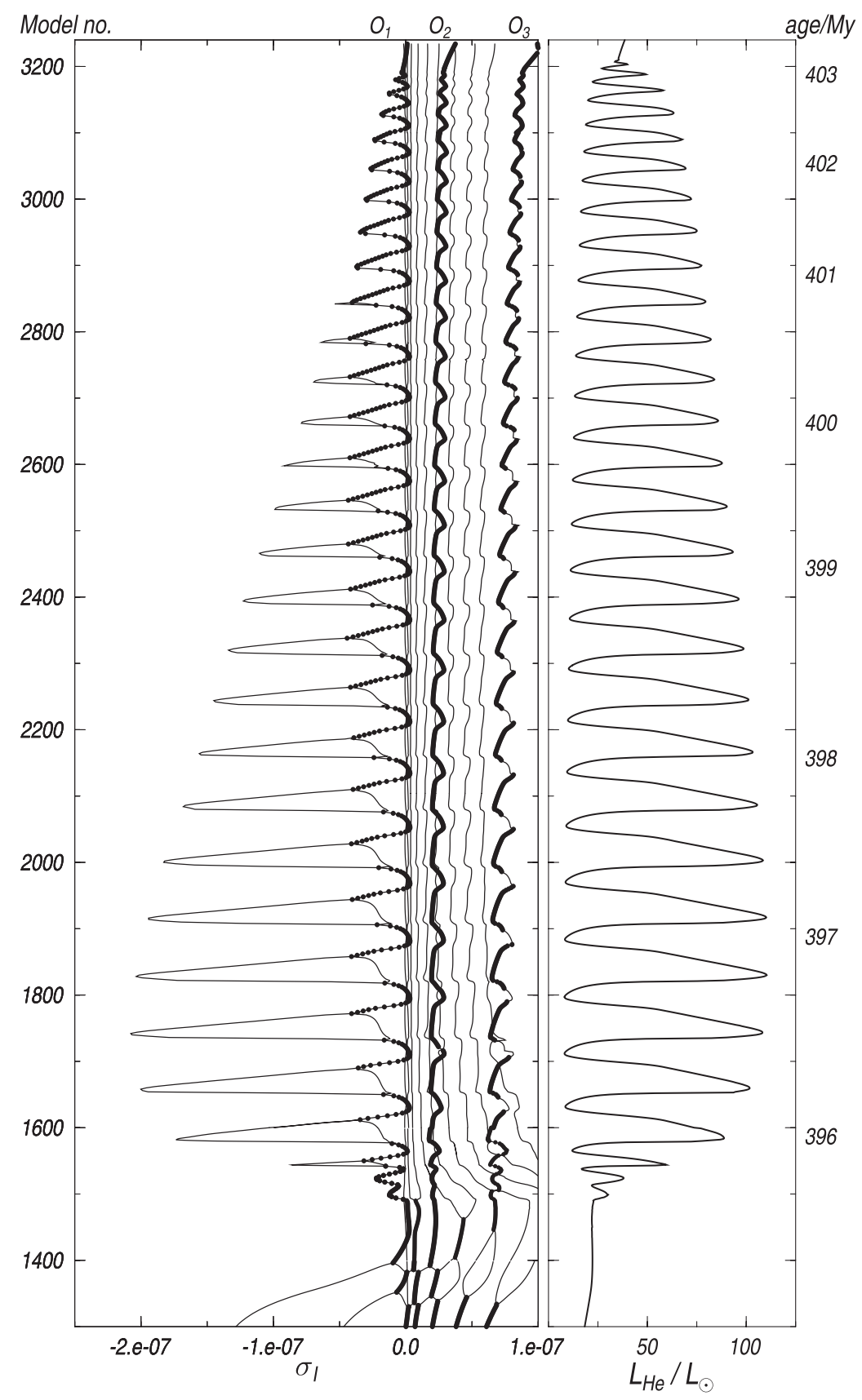

Fig. 4. The left panel of the graph shows the modal diagram with the imaginary parts $\left(\sigma_{\mathrm{I}}\right)$ of the secular modes of the model series presented in Fig. 1. The evolutionary epochs defining the ordinate are counted in model numbers on the left and in mega-years on the right. Oscillatory secular modes, with non-vanishing real parts of the eigenfrequencies, are displayed as filled circles. For reference in the text, the three main branches of oscillatory secular modes extending over most of the ThMP phase are labeled by $\mathrm{O}_{1}$, $\mathrm{O}_{2}$, and $\mathrm{O}_{3}$. To ease the association of model numbers with a characteristic physical quantity of that evolutionary epoch, the panel on the right displays the temporal evolution of the luminosity generated by helium burning. unstable; the growth rates of this monotonic mode are two orders of magnitude smaller than those of the overstable complex secular mode. After model 3216, when the oscillatory secular mode went stable and ThMPs disappeared, the lowest monotonic secular mode remained unstable with an e-folding time of $3.4 \mathrm{My}$ (as a comparison, an evolutionary change of the total luminosity by $5 \%$ takes $3.1 \mathrm{My}$ at this epoch of evolution).

For the set of secular modes that remain monotonic at all epochs after the onset of the ThMPs, the $\sigma_{\mathrm{I}}$ 's all vary in phase during a pulse cycle. Furthermore, the general $\sigma_{\text {I }}$ evolution during the ThMP episode is the same for all these monotonic secular modes; and this evolution differs from the oscillatory secular modes. The latter fact is most evident in the evolution of $\sigma_{\mathrm{I}}$ of the most strongly damped oscillatory mode relative to the monotonic secular modes between models 1500 and about 1800 .

The stellar model sequence whose computation included diffusive overshooting and which showed no evidence at all of thermal pulses in the evolution computations also revealed no overstable oscillatory secular mode at any time during the double-shell nuclear burning phase. However, from the very beginning of the core helium burning through the double-shell burning phase at least one monotonic secular mode was always unstable. Overshooting left its imprints in the abundance structure and in a bigger stellar core as compared with ThMP models.

\subsection{On the suppression of thermal micropulses}

The ThMPs are found to be a delicate phenomenon. First of all, ThMPs are encountered only in computations with appropriate convection treatment and in the narrow mass range between about 2.5 and about $3.5 M_{\odot}$. The easiest, and in some sense the most dangerous way to miss the ThMPs is by means of the timestep choice to evolve a model star. Already Schwarzschild \& Härm (1965) cautioned in their paper that inappropriately large 


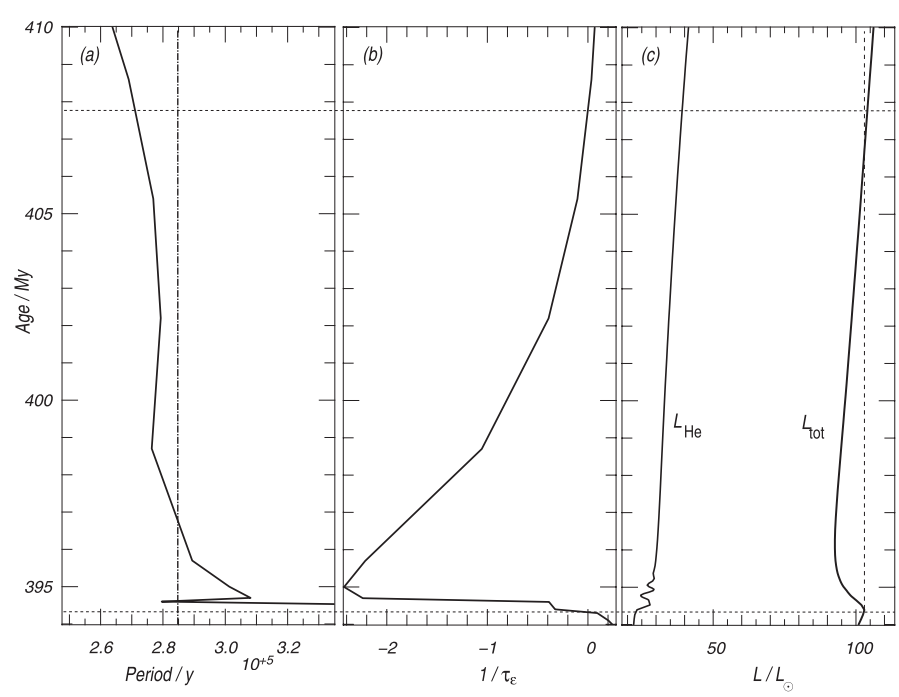

Fig. 5. Panels a) and b) show eigendata of the overstable oscillatory secular mode as computed in the pulse-suppressing model sequence which is characterized in panel c) by the helium-luminosity $\left(L_{\mathrm{He}}\right)$ and the total luminosity ( $L_{\text {tot }}$ ) evolution. Panel a) displays the period evolution of the overstable oscillatory secular mode as a function of model age. The vertical line shows the magnitude of the average pulse-cycle length that was deduced from Fig. 2. The inverse of the e-folding time $\tau_{\mathrm{e}}$ (measured in units of mega-years) of the overstable secular mode is plotted in panel b).

time-steps, of the order of twice the $e$-folding time of the instability, suffice to numerically suppress a pulse. We repeated the computation described in Sect. 3.1 (we refer to it as the "original" one) by enforcing a larger time-step. The coarser-grained time evolution sequence started helium shell burning with a time-step that was 1.88 times larger than that of the original sequence. The structure (position and thickness) of the helium shell and the abundance profiles differed only marginally from those in the original computation. Nonetheless, the helium shell remained essentially stable: we only observed a short initial oscillatory phase of $L_{\mathrm{He}}$ lasting for roughly three strongly damped cycles. The first cycle had the largest amplitude with a relative $L_{\mathrm{He}}$ variation of $11 \%$. (Note that, in the original evolution computation, the relative ThMP amplitude of the helium shell reached $400 \%$.) No variability was manifested in $L_{\text {tot }}$. Even an inspection at the highest numerical resolution of the computed observable stellar parameters revealed no sign of an instability whatsoever (see panel c of Fig. 5).

Figure 5 displays results from computations performed on the pulse-suppressing stellar evolution sequence. As mentioned before, we found one overstable secular mode with characteristics that were comparable to the overstable secular mode in the thermally pulsing model sequence. The dashed horizontal lines extending across all three subpanels of the figure indicate, at the bottom, the onset of the secular instability and the termination of the instability in the upper part of the plot. Panel (a) shows the period evolution of the overstable secular mode as a function of model age; the dash-dotted line in this panel indicates the mean pulse period as derived from the fully pulsing evolutionary sequence. Note that the period obtained in the linear analysis deviates by less than $10 \%$ from the completely independently computed nonlinear cycle length of the pulses. The inverse of the e-folding time (measured in mega-years) of the instability is plotted in panel (b). For most of the instability phase, the e-folding time, $\tau_{\mathrm{e}}$, is less than or of the order of the evolutionary time-scale itself. Panel (c) shows the luminosity evolution of helium burning $\left(L_{\mathrm{He}}\right)$ and of the star's total luminosity, $L_{\mathrm{tot}}$. The quantity $L_{\mathrm{He}}$ of the evolution computation shows only a weak sign of instability that lasts for roughly three strongly damped cycles; the total luminosity carries no sign at all of an instability. We emphasize again that the phase of instability as obtained from the linear secular analysis is comparable to the evolutionary phase over which the ThMPs are encountered in the "non-suppressing" model sequence; this is traced out with the vertical line plotted from the local maximum of $L_{\text {tot }}$ in panel (c). The local maximum at the bottom of the figure coincides with the epoch of model 1490 shown in panel (c) of Fig. 1. In the "non-suppressing" model sequence, the ThMPs are superimposed on the ensuing broad $L_{\text {tot }}$ trough; the luminosity of the terminal model E (whose oscillatory secular modes were all at least weakly stable) lies slightly below the total luminosity of model 1490. In the pulse-suppressing sequence, on the other hand, the linear instability extends to roughly the epoch when the luminosity reaches again the level of the initial bump.

\section{Discussion}

In accordance with Härm \& Schwarzschild (1972) we found the secular eigenspectrum to show an oscillatory secular mode becoming unstable at the epoch of the ThMPs setting in. The secular mode $\mathrm{O}_{1}$ remained purely oscillatory through the first two cycles. Starting with the third cycle, $\mathrm{O}_{1}$ unfolded temporarily into a pair of purely monotonic modes, both of which were unstable. The monotonic-mode interlude took up roughly $46 \%$ of a cycle (at cycle 7) with a slow decrease of this percentage as the ThMP episode progressed. After cycle 21, $\mathrm{O}_{1}$ remained again purely oscillatory up to the last ThMP cycle (number 30 ). It was always around the $L_{\mathrm{He}}$-maximum phases where the oscillatory $\mathrm{O}_{1}$ mode unfolded. Interestingly enough, $\mathrm{O}_{3}$, the other oscillatory secular mode with eigenvalue unfoldings, showed these to occur at around the phases of minimum $L_{\mathrm{He}}$.

Figure 6 shows the periods computed for the mode branch $\mathrm{O}_{1}$ as black continuous lines. The lengths of the ThMP cycles derived directly from the evolutionary computations are plotted as a grey line. The sharp horizontal excursions in the figure are caused by the unfoldings of the complex conjugate eigenvalues and by the merging of two monotonic secular modes where the period goes formally to infinity. The agreement between linear theory and the cycle lengths deduced from the nonlinear stellar evolution is reasonable. The variation of the "linear" period derived from the secular stability analysis during a ThMP cycle is reminiscent of the variation of the pulsation period computed on a nonlinearly pulsating star at various phases of a pulsation cycle (cf. footnote in Sect. 3.2). The open circles denote the periods as measured at phases with $L_{\mathrm{g}}=0$, i.e. at formal equilibrium phases. The maximum deviations of the cycle lengths derived from the evolutionary computations and the periods at equilibrium phases do not exceed $40 \%$. The correspondence of the two differently computed cycle lengths in Fig. 6 are considered as a good indication that a linear secular analysis is appropriate to at least qualitatively understand the cyclic variability of the ThMP phase; this point of view is further supported by the results of the secular analyses of the pulse-suppressing model sequence which was presented in Sect. 3.3. The periods obtained in the pulse-suppressed series agree very well with the cycle lengths measured in the evolution computations and are well bracketed by the secular analyses of the thermally pulsing models.

The discussion is structured as follows: first, we comment on the local criteria for secular instability and the difficulty of their 


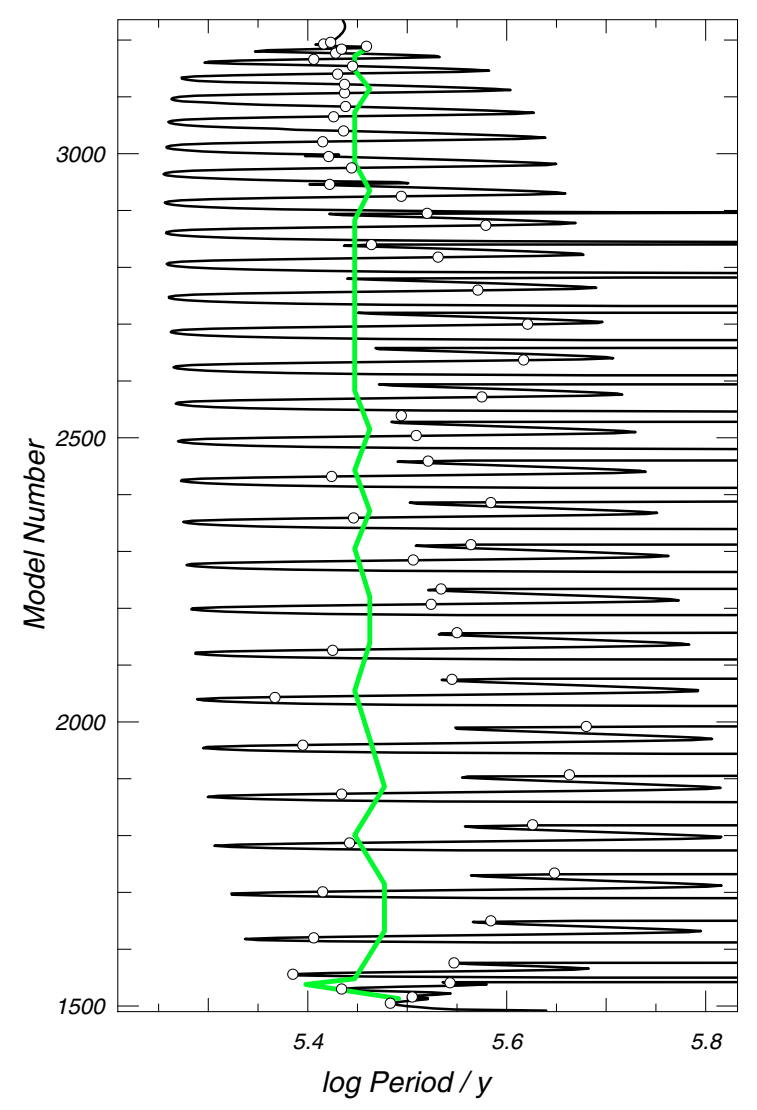

Fig. 6. The periods of the overstable oscillatory secular mode $\mathrm{O}_{1}$ shown in Fig. 4 during the ThMP episode as a function of model number. The almost horizontally running lines result from the unfolding and merging of secular modes. At the corresponding phases, the periods go formally to infinity. The thick grey line indicates the cycle lengths that were derived directly from the $L_{\mathrm{He}}$ variation of the evolutionary computations. The open circles indicate the periods as measured at pulse phases with $L_{\mathrm{g}}=0$, i.e. at "equilibrium" epochs.

use in case of oscillatory secular modes. A short digression on the attempts to understand what discriminates between monotonic and oscillatory secular modes follows. We then delve into the properties of the linear eigenmode spectrum as we computed it. We close by checking the quality of the Gabriel-expansion during the short-term evolution of the ThMP phase.

\subsection{Local stability criteria}

The paper of Schwarzschild \& Härm (1965) contains a simple conceptual model of a nuclear-burning shell in a star. The formulae they obtained at the end of their local analysis allow a quick estimation of the shell's stability against thermal perturbations; therefore, the local criteria have been used ever since in computational research and in textbooks. It turns out that two conditions must necessarily be fulfilled for instability: the shell must be thinner than

$\frac{\Delta r}{r}<\frac{\Gamma_{1}}{4} \cdot f, \quad$ but sufficiently thick so that $\quad \frac{\Delta T}{T}>\frac{4}{\varepsilon_{T}}$,

the quantity $f$ measures the deviation from a homologous perturbation; most frequently $f$ is chosen ad hoc to be about unity, i.e. close to homology. The variation of physical quantities across the nuclear burning shell are indicated with a leading $\Delta$. The width of the shell is not uniquely defined and gives rise to considerable ambiguity. The remaining quantities take on appropriate
Table 1. Evaluation of physical quantities across the He-shell.

\begin{tabular}{cccccc}
\hline \hline Model & Width & $\Delta r / r$ & $\Gamma_{1} / 4$ & $\Delta T / T$ & $4 / \varepsilon_{T}$ \\
\hline \multirow{7}{*}{1470} & $\varepsilon_{\max } / 10$ & 0.122 & 0.41 & 0.067 & $0.1-0.09$ \\
& $\varepsilon_{\max } / 100$ & 0.23 & 0.41 & 0.129 & $0.1-0.09$ \\
& $\varepsilon_{\max } / 1000$ & 0.318 & 0.41 & 0.168 & $0.1-0.08$ \\
1516 & $\varepsilon_{\max } / 10$ & 0.175 & 0.41 & 0.067 & $0.11-0.09$ \\
& $\varepsilon_{\max } / 100$ & 0.258 & 0.41 & 0.109 & $0.11-0.09$ \\
& $\varepsilon_{\max } / 1000$ & 0.349 & 0.41 & 0.149 & $0.11-0.08$ \\
3234 & $\varepsilon_{\max } / 10$ & 0.413 & 0.41 & 0.149 & $0.32-0.11$ \\
& $\varepsilon_{\max } / 100$ & 0.514 & 0.41 & 0.206 & $0.32-0.13$ \\
& $\varepsilon_{\max } / 1000$ & 0.585 & 0.41 & 0.224 & $0.32-0.08$ \\
\hline
\end{tabular}

mean values that are representative of the nuclear-burning shell. With $\varepsilon_{T}$ we denote $\partial \log \varepsilon / \partial \log T$ at constant pressure. The remaining quantities have their canonical meanings. Note that the instability that is described with conditions (1) is a monotonic one; this simple one-zone model does not allow for complex solutions at all. Therefore, relying on criteria (1) in the case of thermal-pulse cycles that start via oscillatory secular modes can be misleading.

Table 1 contains numerical values of the two sides of both instability criteria in (1) for selected stellar models and for various choices of the width of the He-burning shell. The thickness of the shell was measured as the extension over which nuclear energy generation exceeds a prescribed fraction of $\varepsilon_{\max }$. The range of numbers given in column " $4 / \varepsilon_{T}$ " is caused by the non-negligible variation of $\varepsilon_{T}$ across the nuclear burning shell. Model 1470 was secularly stable, it showed no unstable secular modes and around that epoch $L_{\mathrm{He}}$ varied still only weakly and monotonously. At model number $1516, L_{\mathrm{He}}$ just passed through the first peak and mode $\mathrm{O}_{1}$ was overstable at this epoch; the ThMP-phase just developed. Finally, model number 3234 lies after the ThMP phase with no overstable oscillatory secular modes anymore and $L_{\mathrm{He}}$ changed again slowly and monotonously.

Most frequently, only the first condition of the instability criteria (1) is referred to in the literature; i.e. only the test if a shell is sufficiently thin for the instability to develop. The instability condition in expressions (1) is equivalent to the physical notion of a generalized specific heat (also referred to as "gravothermal" specific heat) which becomes positive within the nuclear burning shell. Giannone \& Weigert (1967) generalized the local instability criteria (1), adopting a more general equation of state and allowing for more variation of the physical background quantities across the nuclear-burning shell. Yoon et al. (2004) elaborated on the local stability criteria of nuclear burning shells; building upon the approach of Giannone \& Weigert (1967) but still with the simplifying assumption of homologous perturbations. The aim was to quantitatively test stellar evolution models towards thermal-pulse instabilities. The problem with the local stability criteria lies mainly in the necessity to have to assume a functional form of the perturbations (usually homologous motion) and therefore to constrain the nature of the instability and to have to adopt a not well definable thickness of the shell. Yoon et al. (2004) mention that the shell thickness in their helium-accreting white dwarf changes by about $15 \%$ if they use the radii once at $r\left(\varepsilon=10^{-2} \varepsilon_{\max }\right)$ and once at $r\left(\varepsilon=10^{-3} \varepsilon_{\max }\right)$ with $\varepsilon_{\max }$ being the peak nuclear burning rate. For their discussions, Yoon et al. chose $r\left(\varepsilon=2 \times 10^{-3} \varepsilon_{\max }\right)$ as their local analyses agreed best with the evolutionary computations. This approach might be incomplete, however, since, as we have discussed before, the dissipation inherent in the computational schemes for stellar 
evolution might suppress thermal pulses despite their being physically present and detectable in a full linear secular analysis.

In Table 1, local secular instability obtains if the numbers of column " $\Delta r / r$ " are smaller than those of column " $\Gamma_{1} / 4$ " and if the numbers of column " $\Delta T / T$ " are bigger than those of column " $4 / \varepsilon_{T}$ ". For model 1470 , the geometrical criterion in (1) indicates instability for all choices of shell thickness; the thermal part of the local condition favors instability for sufficiently thick shells (i.e. for $\varepsilon_{\max } / 100$ and $\varepsilon_{\max } / 1000$ ). Note that for model 1470 (cf. Fig. 4) neither the solution of the full secular boundary-value problem nor stellar evolution shows a sign of secular instability yet. For model 1516, the geometric and the thermal local criteria behave the same as for model 1470. In this case, it is compatible with stellar evolution and full secularanalysis results. For model 3234, at an epoch in the post-ThMP phase, all choices of shell size point to stability and, depending on the choice of a representative magnitude of $4 / \varepsilon_{T}$, the same applies to the thermal criterion.

In agreement with Yoon et al. (2004) we find that the particular choice of shell thickness influences crucially the result of the local criterion. Moreover, the onset of the ThMPs is not captured reliably with the local stability criteria. This does not surprise further as the ThMPs are the result of the instability of a complex secular mode and the local criteria are based on a onezone model description that do not support complex solutions at all.

In the thermal criterion of conditions (1), thickness is expressed as temperature and luminosity differences across the burning shell, these quantities are more sensitive to a particular choice of shell-boundaries than the radius measures; therefore the thermal criterion is more sensitive to a particular choice of shell thickness.

\subsection{Oscillatory versus monotonic secular solutions}

Already Gabriel \& Noels (1972) stressed that due to the nonhermiticity of the secular stability problem, complex eigenvalues must not be considered unusual. An unambiguous prescription of conditions that must prevail in a star to lead to complex eigensolutions have not been put forth in the literature so far.

As mentioned at the beginning of Sect. 4, the merging/unfolding phases of the $\mathrm{O}_{1}$ mode branch during the pulse cycles differ from that of $\mathrm{O}_{3}$ (see Fig. 4); this immediately enforces the important conclusion that stellar background quantities alone do not discriminate between monotonic and complex secular eigensolutions, an aspect already conjectured by Defouw (1973). Despite adopting the point of view that the thermal pulses themselves are (nonlinear) relaxation oscillations, Defouw (1973) emphasized the combination of hydrostatic readjustment to a heat perturbation and the magnitude of the temperature dependence of nuclear burning, $\varepsilon_{T}$, to decide over the nature of the secular eigenvalue. Earlier, Dennis (1971) studied the dependence on $\varepsilon_{T}$ of the secular stability of the helium-burning shell of massive stars. Low values of $\varepsilon_{T}$, left the considered secular mode oscillatory damped; increasing $\varepsilon_{T}$ drove it overstable and for $\varepsilon_{T}>53$, it eventually unfolded into two monotonic branches. In our computations, we found the magnitude of $\varepsilon_{T}$ at the peak of the unstable He-burning shell to stay at about 40 and to vary by less than 5\% during the ThMP cycles; in the case of the unfolding and re-merging of the $\mathrm{O}_{1}$ mode, this variation was furthermore opposite to what Dennis (1971) suggested for this process. Hence, together with the phase-shifted merging/unfolding behavior of $\mathrm{O}_{3}$, we conclude that the importance of $\varepsilon_{T}$ lies at best in its magnitude but not in its variation.

\subsection{On the secular-eigenmode spectrum}

Looking at the modal diagram in Fig. 4, we realize that the oscillatory and the monotonic secular modes evolve differently on the $\sigma_{\mathrm{I}}-$ age plane. This applies also to cyclically repeating episodes of unfolding of complex eigenmodes into two monotonic modes during the ThMP phase and in particular it is true for the early ThMP phase when the He shell source takes over from central helium burning. However, throughout the whole ThMP phase the stable oscillatory secular modes $\left(\mathrm{O}_{2}\right.$ and $\left.\mathrm{O}_{3}\right)$ show an average $\sigma_{\mathrm{I}}$ increase that exceeds that of the monotonic modes; this suggests that oscillatory and monotonic secular modes are each influenced by different regions of the stellar interior. Unfortunately, the secular eigenproblem displays no special mathematical structure; in particular all the helpful tools developed for the hermitian adiabatic pulsation problems do not grip. We must therefore resort to analyze eigenfunctions at selected instances and hope to derive thereof useful hints on the nature of the secular modes.

In the following, we refer to the eigenfunction components $\zeta$, $p, t$ and $\delta q \equiv T \delta s$ standing for the relative radius, pressure, temperature, and heat perturbation, respectively. We studied in more detail the eigenfunctions at the begin of the ThMP episode: For model 1488, just before the onset of the thermal micropulses, for model 1492, one stellar model after the onset of the micropulses, and for model 1516, at the $L_{\mathrm{g}}=0$ phase after the first pulse cycle. Since, as mentioned before, the secular problem does not display any favorable mathematical structure, we cannot assume any ordering of the modes (e.g. no correlation of node numbers and the magnitude of the eigenfrequency) and indeed this is what we encountered. The counting was exemplarily performed on eigenfunctions of the lowest five monotonic eigenmodes, and the first three oscillatory ones $\left(\mathrm{O}_{1}, \mathrm{O}_{2}, \mathrm{O}_{3}\right)$. Node counting revealed that the mechanical eigenfunction components and the thermal ones behave differently. For all modes considered, the mechanical eigenfunction components had either 2 or 3 nodes; i.e. the number of nodes of the displacement and the relative pressure perturbation was independent of the particular mode. Furthermore, the number of nodes of a chosen mode was subject to change (by one or two nodes) as the star model evolved. For all secular modes, monotonic and oscillatory, the radial displacement admits one node either within or close to the hydrogen burning shell.

Figure 7 plots representative eigenfunctions of mode $\mathrm{O}_{1}$ in model 1516. The top panel shows the spatial variation of the phase of the heat perturbation $\delta q$. The middle panel displays the real parts of $\zeta, t$, and of the suitably scaled $\delta q$; the corresponding imaginary parts of the eigenfunctions are contained in the bottom panel. For better orientation, the middle panel is complemented with a dash-dotted line tracing the (again suitably scaled) spatial variation of the star's luminosity. The node of $\zeta$ at the H-burning shell is reminiscent of the "mirror principle" encountered in the spatial variation of the radius evolution of many shell-burning stars. Only mode $\mathrm{O}_{1}$ has a relatively large displacement amplitude also interior to the helium burning shell. The displacements of modes $\mathrm{O}_{2}$ and $\mathrm{O}_{3}$ become marginal already below the hydrogen-burning shell. On the other hand, many of the monotonic modes which we studied have non-negligible displacement amplitudes in the deep interior with these amplitudes growing with $\left|\sigma_{\mathrm{I}}\right|$. Also the monotonic secular modes show a node close to the H-burning shell. The outermost node lies in the envelope with its position in radius to grow with $\left|\sigma_{\mathrm{I}}\right|$, for all these monotonic modes, the maximum amplitude is reached at the surface. For all modes considered, the perturbations 


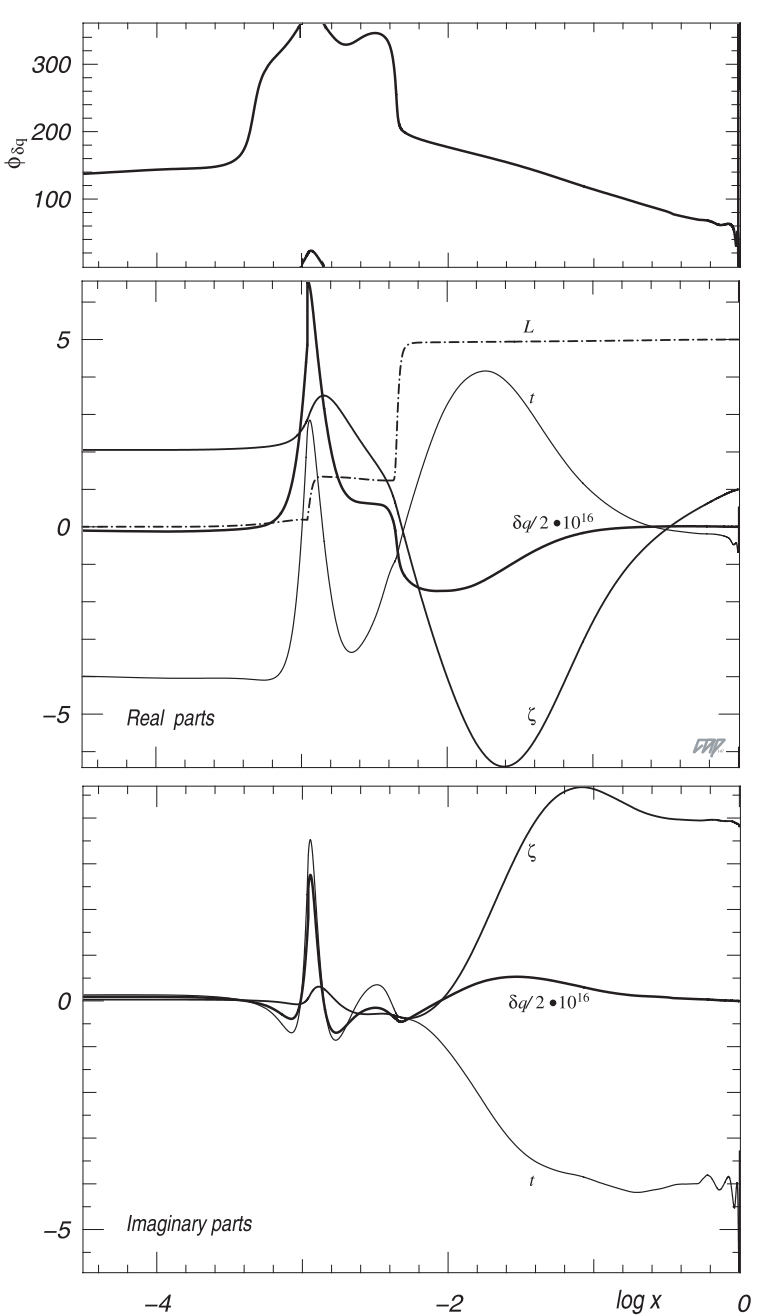

Fig. 7. Spatial run, measured in fractional radius $x$, of eigenfunction data of the $\mathrm{O}_{1}$ mode in model 1516. The two lower panels show the real and imaginary parts of the relative temperature perturbation $t$, the radial displacement $\zeta$, and the heat perturbation $\delta q$. The heat perturbation and the luminosity $L$ (dash-dotted line in the middle panel) of the stellar model are suitably scaled to fit the plot range. The top panel shows the spatial variation of the complex phase angle of the heat perturbation $\delta q$.

become homologous below $\log x \approx-3.5$; i.e. homologous variation prevails only over a tiny fraction of the star's radius and in particular it does not obtain at and above the nuclear burning shells. Focusing on the perturbations of thermal quantities revealed that e.g. $\delta q$ of the oscillatory secular modes $\left(\mathrm{O}_{1}\right.$ to $\left.\mathrm{O}_{3}\right)$ is considerably confined to the intershell region of the stellar models with noticeable amplitude at the base of the star's envelope, just above the hydrogen shell. In contrast, the heat perturbations of the monotonic modes achieve their largest amplitudes in the very deep stellar interior with the heat perturbations in the nuclear-burning shell region being relatively small. Based on the behavior of the thermal perturbations, we can consider the oscillatory modes as functionally different from the monotonic ones. Furthermore, the thermal eigenfunctions show a node behavior that differs from that of the mechanical ones. The number of nodes of the thermal components exceeds that of the mechanical ones in all models considered. In contrast to the mechanical eigenfunctions, the thermal eigenfunctions of different mode branches always have different numbers of nodes (e.g. at model 1488: 4 nodes in $\delta q$ for $\mathrm{O}_{1}, 7$ for $\mathrm{O}_{2}$, and 11 for $\mathrm{O}_{3}$ ). But again, as for the mechanical eigenfunctions, the number of nodes of the thermal eigenfunctions of a particular mode branch is not constant as the model evolved.

It is worthwhile pointing out that, in contrast to the findings of Härm \& Schwarzschild (1972), the imaginary parts of the eigenfunctions of the oscillatory modes are always comparable to the real parts over most of the stellar interior; this applies for the mechanical quantities as well as for the thermal variables (cf. Fig. 7). The phase of $\delta q$ varies significantly over most of the star's radius, in particular the envelope does not vary synchronously; a fact which is quantified in the top panel of Fig. 7. The most rapid phase variations occur in the H-burning and just below the He-burning shell. Across the helium-burning shell, the phase stays essentially constant, so that eigenfunctions behave, at least locally, like standing waves. And as is to be expected, in the region where the perturbations are homologous, i.e. for $\log x<-3.5$, the phase variation levels off.

The phase variation of $\delta q$ is also relevant for another aspect: Härm \& Schwarzschild (1972) postulated without further specification that the complexification of secular eigenfrequencies relies on the coupling of a "reacting" and a "driving" layer, with $\delta q$ being $\pi / 2$ out of phase in the two layers. The reacting layer was identified with a region just inside the He-burning shell; stellar material above the helium shell was thought to have too low a heat capacity to constitute a pertinent reactive layer and was therefore discarded. But only the two-zone models (TZM) of Defouw (1973) eventually characterized the physical requirements for the occurrence of complex eigenfrequencies in the secular problem. The TZM were successfully reproducing results of previous numerical experiments which aimed at understanding the occurrence of oscillatory secular modes.

To quantify the phase difference between reacting and driving region we resort here to the "ultra-simplified model" which neglects the thermal coupling between the two zones (cf. Sect. VI of Defouw 1973); in Defouw's notation the phase angle $\phi$ between the heat perturbations of the TZM can be written as $\tan \phi=-n_{I} /\left(\lambda_{1} c_{11}-n_{R}\right)$. Hence, a phase difference of $\pi / 2$ (as referred to in Härm \& Schwarzschild 1972) between the driving and the reacting zone is recovered only for the very special circumstance of $\lambda_{1} c_{11}-n_{R}=0$; i.e. for $n_{R}=0$ and $c_{11}=0$ which means for a neutral oscillation (temporal variation $\propto \exp (n \cdot t)$ ) of a model with an infinite gravothermal specific heat of the driving region, or for the very special correlation $\lambda_{1} c_{11}=n_{R}$. In all other cases, however, $0<\phi<\pi / 2$ between $\delta q$ of the driving and reacting regions, depending on $n_{R} / n_{I}$ and $\lambda_{1} c_{11} / n_{I}$. Together with the fact that the heat capacity of the intershell region is not lower than that of the He-shell, which is considered the driving region, we conclude that it is not unreasonable to contemplate the intershell region or part thereof as the reacting layer. Furthermore, the differential work plot shown in Fig. 8 hints at a higher reactivity of the intershell region than of the stellar layers on the inside of the He shell.

The lower two panels of Fig. 7 display the heat perturbation $\delta q$ as a heavy line and the relative temperature perturbation, $t$, as a thin solid line. The displayed spatial run is representative of the $\mathrm{O}_{1}$ behavior during the whole ThMP phase. We emphasize the positive correlation between $\delta q$ and $t$ in the He-burning shell: locally a necessary condition for a secular instability to develop; whether a star becomes eventually unstable depends of course on the detailed damping and excitation efficiencies throughout the whole object. (The amplitude of $\delta q$, an absolute perturbation, was divided ad hoc by $2 \times 10^{16}$ to match the scale of the figure.) If $\delta q=A \cdot \delta T / T$ with $A>0$ lies in nuclear-burning layers with sufficiently temperature-sensitive reactions, this helps for 


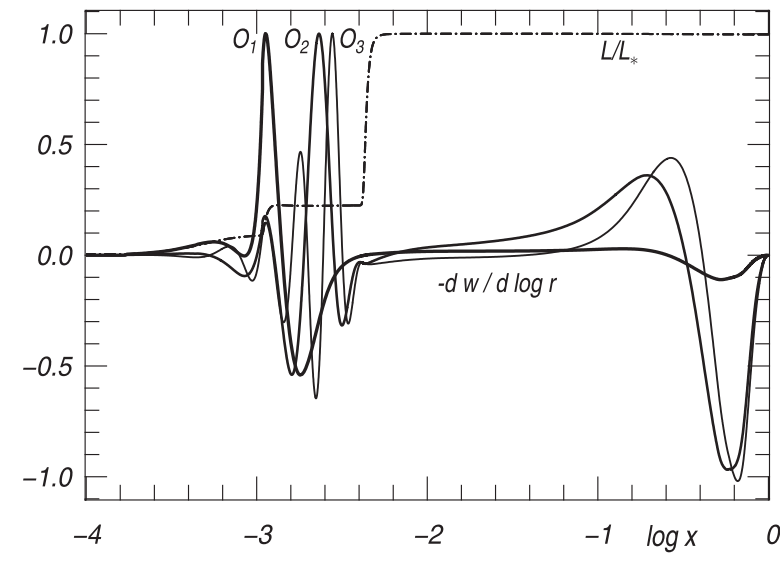

Fig. 8. Differential work integrals $-\mathrm{d} w / \mathrm{d} \log r$ throughout a star's interior. The sign convention is so as to show driving regions positive and damping ones negative. The dash-dotted line depicts, to help orientation, the spatial variation of the total relative luminosity, $L / L_{*}$. The data is shown for model 1492, the epoch just after the onset of the thermal micropulses with mode $\mathrm{O}_{1}$ having gone overstable.

a marginal heat perturbation to develop into an instability. The $\delta q=A \cdot \delta T / T$ correlations in suitable stellar layers were encountered only for $\mathrm{O}_{1}$ modes. The other oscillatory secular modes, despite also having positive $\delta q-\delta T / T$ correlations, these correlations were confined to the intershell region where no physical agent exists to exploit the temperature perturbation at the appropriate phase. Also the monotonic modes show regions of suitable $\delta q-\delta T / T$ correlations which were usually located in the very deep interior, below the He-burning shell, i.e. in the inert $\mathrm{C} / \mathrm{O}$ interior.

To more directly illustrate the action of the helium shell to power the instability and thermal activity of the intershell region we computed work integrals for some overstable oscillatory secular modes. Following the reasoning and interpretations of Glatzel (1994), we justify the adoption of the work integral even in the case of rapidly growing instabilities.

For model 1492, Fig. 8 displays the differential work curves

$-\frac{\mathrm{d} w}{\mathrm{~d} \log r} \propto \operatorname{Im}\left(t^{*} p\right) P r^{3} \delta$

of the oscillatory modes $\mathrm{O}_{1}$ to $\mathrm{O}_{3}$. We defined $\delta \equiv$ $\partial \log \rho / \partial \log P$ at constant temperature. The asterisk refers to the complex conjugate of the quantity to which it is applied. All differential-work curves in Fig. 8 are normalized independently to unity at the positions of their respective maximum driving as we are mainly interested in illustrating the domains of "activity". The spatial variation of the differential work is representative of each mode for the whole ThMP episode; it is only the strengths of the various driving and damping regions that change relative to each other. For better orientation, the thin dash-dotted line in Fig. 8 traces out the spatial run of the total relative luminosity. For the $\mathrm{O}_{1}$ mode, it is evident that the maximum driving occurs in the helium-burning shell. The intershell domain between helium and hydrogen burning constitutes the dominant damping region. Some minor additional damping is observed in the outermost layers. As the ThMPs begin, the driving in the Heburning shell overcompensates the intershell damping. Finally, as the star stabilizes secularly, the driving and damping features persist as before; however, the damping regions eventually win over the weakening driving effect in the fattening He shell. For $\mathrm{O}_{2}$ and $\mathrm{O}_{3}$, only weak driving occurs in the He-burning shell,

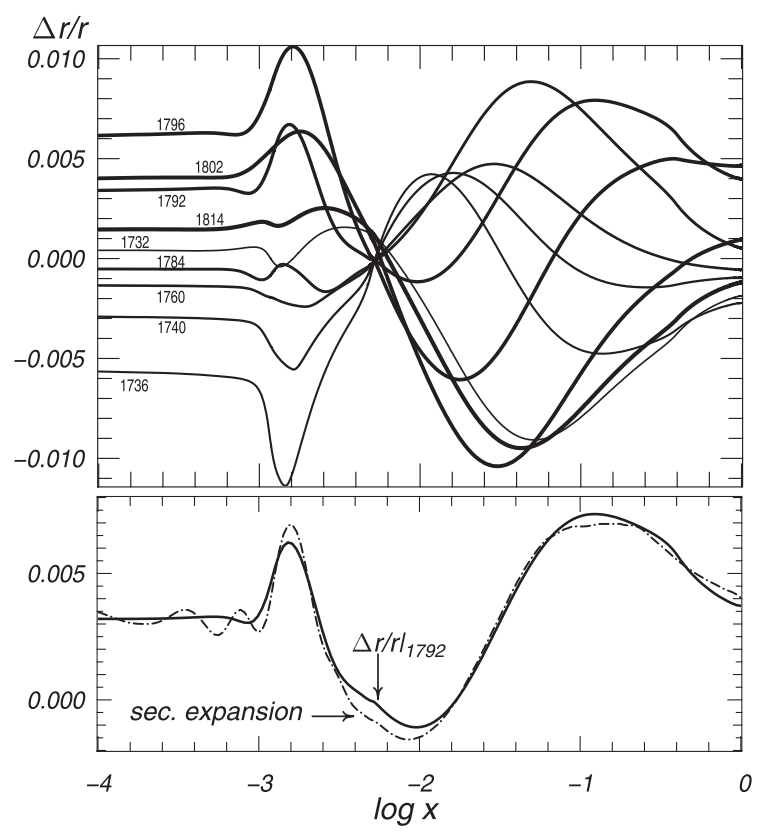

Fig. 9. The top panel shows the relative radius change, $\Delta r / r$, as a function of the relative stellar radius $x$ derived from the stellar evolutionary sequence, as the star evolves through the ThMP-cycle between model numbers 1732 and 1814. This phase covers the largest-amplitude modeunfolding episode depicted in Fig. 4. The bottom diagram compares the quantity $\Delta r / r$ at model 1792 with the result of the Gabriel-expansion with 9 terms, applied to the displacement eigenfunction.

the intershell region exhibits almost canceling driving and damping. It is then the envelope that eventually determines the fate of the modes' stability with strong damping throughout most of the outer layers.

Hansen (1972) pointed out the running thermal-wave character of oscillatory secular modes. Studying the temporal variation of the complex eigenmodes in our models indeed revealed a pronounced propagative behavior. All complex eigensolutions behave similarly in that outward propagating patterns develop in the inter-shell region; the He-burning shell acts as a - spatially stationary - piston for these features. Below the He shell, heat perturbation and radial displacement behave mostly like standing waves with only a weak inward-running component.

\subsection{The relevance of the secular modes on the star's evolution}

At the end, we want to find out how well the expansion of Gabriel (1972) does in the context of our ThMP problem. We address this aspect with the help of Fig. 9; its top panel shows the spatial variation of $\Delta r / r=1-r\left(m, t_{0}\right) / r\left(m, t_{1}\right)$ (with $t_{0}, t_{1}$ denoting temporally neighboring models) during the ThMP cycle which is bounded by models 1732 and 1814 - this cycle encompasses the largest-amplitude mode-unfolding seen in Fig. 4. The model numbers given in Fig. 9 refer to the later epochs used to compute $\Delta r / r$; the bump at about $\log x=-2.8$ (location of the He-shell) is spatially an essentially stationary feature, the same applies to the homologous radius variation of the even deeper lying regions. An almost perfect node (at $\log x \approx-2.2$ ) lies at the base of the hydrogen-burning shell. This node is a manifestation of the "mirror principle" which is referred to frequently in the context of shell burning stars. Finally, an outward propagating bump develops above the hydrogen-burning shell; its 
Table 2. Coefficients of the expansion of the evolutionary $T \mathrm{~d}_{t} s$ in terms of secular eigenmodes for model 1792. The first column gives the number of terms of the series expansion. The modes used in the series are denoted by O's for the oscillatory ones (cf. Fig. 4) and M's for monotonic secular modes. The second lines in the columns of $\mathrm{O}_{1}$ and $\mathrm{O}_{2}$ give the imaginary parts of the respective expansion coefficients.

\begin{tabular}{cccccccccc}
\hline \hline Number of modes & $\mathrm{O}_{1}$ & $\mathrm{O}_{2}$ & $\mathrm{O}_{3}$ & $\mathrm{M}_{1}$ & $\mathrm{M}_{2}$ & $\mathrm{M}_{3}$ & $\mathrm{M}_{4}$ & $\mathrm{M}_{5}$ & $\mathrm{M}_{6}$ \\
\hline 3 & 0.177 & 0.301 & -0.031 & & & & & & \\
& 0.427 & 0.124 & & & & & & & \\
4 & 0.238 & 0.355 & -0.085 & -0.289 & & & & & \\
& 0.513 & 0.270 & & & & & & & \\
5 & 0.223 & 0.331 & -0.074 & -0.289 & 0.003 & & & & \\
& 0.494 & 0.301 & & & & & & & \\
6 & 0.264 & 0.363 & -0.087 & -0.319 & 0.012 & -0.006 & & & \\
& 0.536 & 0.236 & & & & & & & \\
7 & 0.248 & 0.331 & -0.049 & -0.293 & 0.014 & -0.012 & -0.034 & & \\
& 0.456 & 0.360 & & & & & & & \\
8 & 0.209 & 0.105 & -0.012 & -0.278 & 0.012 & -0.009 & -0.012 & 0.126 & \\
& 0.387 & 0.474 & & & & & & & \\
9 & 0.208 & 0.158 & -0.009 & -0.274 & 0.012 & -0.009 & -0.016 & 0.084 & -0.016 \\
& 0.383 & 0.481 & & & & & & & \\
\hline
\end{tabular}

propagation into the star's envelope can be traced even in the time-series of $\Delta r / r$ in the top panel of Fig. 9.

Gabriel (1972) showed that for sufficiently short time-scales, over which a star's evolutionary variations can be linearly approximated relative to a reference epoch, the physical quantities can be expanded into a series of secular eigenfunctions (see Eq. (13) in Gabriel 1972). We followed Gabriel's procedure to find out how well the linear eigensolutions approximate the variation induced by the star's short-term evolution. At (between 100 and 300) equally-spaced gridpoints in $\log x$, we solved the leastsquare problem for the coefficients of the series expansion in $T \delta s$ (cf. Eq. (15) in Gabriel 1972). Table 2 lists the results for model 1792 for various choices of terms included in the series (first column). For each number of modes included in the series expansion, the sum of the coefficients was normalized to unity separately. The mode designated by $\mathrm{O}_{3}$ was unfolded at model 1792 so that its expansion coefficient is real and not complex as for $\mathrm{O}_{1}$ and $\mathrm{O}_{2}$. The monotonic modes which were included in the series expansion are labeled as $\mathbf{M}_{i}$. In contrast to Gabriel's gravitationally contracting model stars, our ThMP models do not show an equally rapid convergence of the series as a function of the number of modes (i.e. terms) included. In accordance with the eigenmode properties discussed in Sect. 4.3 we found modes $\mathrm{O}_{1}$ and $\mathrm{O}_{2}$ to be essential to approximate well the functional behavior in the nuclear burning shells; to also match the core and the envelope variation, the inclusion in the series expansion of (damped) monotonic modes, in particular of $\mathrm{M}_{1}$, turned out to be indispensable; however, at the price of added spatial small-amplitude oscillations in $-4<\log x<-3$. An impression of the quality of the result of Gabriel's procedure applied to $\Delta r / r$ of model 1792 with 9 terms in the series expansion can be obtained from the bottom panel of Fig. 9.

The Gabriel - expansion turned out to do reasonably well to approximate the spatial variation of perturbed physical quantities (see bottom panel of Fig. 9). However, in contrast to many stellar pulsation problems, linear secular theory failed to reproduce the amplitude evolution of the variability throughout the ThMP episode. This is not further surprising as this aim lies outside the domain of validity of the Gabriel-expansion. Nevertheless, the period of the overstable secular mode during the ThMP phase provides a good approximation to the pulse-cycle length of the fully nonlinear problem.
Finally, it is worth emphasizing that the secular problem is strongly non-normal. As such, we cannot, in general, expect a close match of the linear results with the nonlinear developments. For non-normal operators, considerable transient phases are usually encountered at the onset of instabilities, so that normal-mode eigenanalyses might have only limited predictive power (e.g. Trefethen \& Embree 2005; Eisenman 2005). In our case here, however, the agreement is surprisingly good.

\section{Conclusions}

A large number of $3 M_{\odot}$ stellar models that exhibit thermal micropulses at the onset of their double-shell nuclear burning phase were studied by means of linear secular eigenanalyses. The micropulses are considered as physically comparable with the larger-amplitude thermal pulses of low- and intermediatemass stars usually encountered along the advanced AGB. Our computations showed that the ThMP episode sets in via the instability and finally dies away via the restabilization of an $o s-$ cillatory secular eigenmode. And most importantly, the overstability of the lowest-frequency oscillatory secular mode persists over most of the ThMP phase. During the numerous ThMP cycles, the oscillatory modes unfold temporarily into pairs of purely monotonous secular modes. This phenomenon, we think, is the result of performing stability analyses on the actually pulsing stellar models. We conclude that the ThMPs are the nonlinear development of the linear instability of the oscillatory $\mathrm{O}_{1}$ mode. Most importantly, the cyclic nature of the ThMPs has its origin in an unstable linear oscillatory secular mode that persists throughout the ThMP episode; it is therefore not surprising that the linear periods (cf. Fig. 6 and in particular Fig. 5) correlate well with the lengths of the nonlinear pulse cycles. The view of Defouw (1973) of the thermal pulses as being nonlinear relaxation oscillations of monotonic type appears to have been premature as it probably relied on the insufficiently long simulation computations of Härm \& Schwarzschild (1972). Had the latter authors have had at their disposal data covering more thermalpulse cycles, we are confident that they would have observed a re-merging of the two monotonic mode branches into a complex pair during later phases of pulse cycles with sufficiently low amplitude (cf. Fig. 4). The linear aspect of the instability which was emphasized in this paper is not capable of capturing the nonlinear, finite-amplitude development of the various 
physical quantities during the ThMPs. Indications that nonlinear processes are indeed important are hinted at in Fig. 3 which shows that the amplitudes of the radius and density variations in the nuclear-burning shell centers saturate after about the first four ThMP cycles and in Fig. 4 with the amplitude of $L_{\mathrm{He}}$ decreasing slowly after about 397 My despite the persistent overstability of mode $\mathrm{O}_{1}$ until about $403 \mathrm{My}$.

The explanation of thermal pulses as the nonlinear development of a linearly overstable secular mode also leads to a natural explanation of the origin of the core-mass - interflash-period relation which was derived along the advanced AGB (Paczyński 1975). We conjecture that the interflash-period is the period of the overstable secular mode which gets shorter with increasing core mass. Hence, the core-mass - interflash-period relation is actually a secular period - luminosity relation. Unfortunately, the evolutionary phase of the ThMPs is so short that the core mass does not change significantly; therefore, our conjecture could not yet be underpinned quantitatively. Nevertheless, Fig. 5 hints at a decreasing period of the overstable secular mode as the star model ascends the AGB. A detailed study of thermally pulsing AGB stars remains to be done.

Well known, but largely ignored is the fact that stellarevolution results depend, partly even sensitively, on the timestepping of their computation. As known for a long time (e.g. Rose 1966), we could essentially suppress the pulses when adopting even a moderately large time-step (a factor less than two compared with the pulse-resolving time-step). The nonlinear "pulse-suppressing" evolution computation showed a very weak signature in $L_{\mathrm{He}}$ of three strongly damped thermal pulses only. The linear stability analysis on these models continued, on the other hand, to reveal an unstable oscillatory secular mode with the instability extending over essentially the same evolutionary phase as found in the "non-suppressing" computation. Hence, the stellar evolution equations as discretized in most computer codes are inherently dissipative and are prone to suppressing secular instabilities at large time-steps. Therefore, depending on the particular goal, it is not advisable to aim for evolving stars with a minimum of computational time-steps; it also seems dangerous to estimate the stability of shell sources via local criteria alone in stellar-evolution computations (cf. Sect. 4.1).

The possibility of deciding observationally if ThMPs are realized in nature is slim; the luminosity variations during the pulses being very small and on much too long a time-scale to be potentially detectable in flagranti. Furthermore, the number of stars in the appropriate evolutionary stage in a stellar aggregate such as a cluster seems too small to be observable via an accumulation effect in say a color-magnitude diagram. Last but not least, we found no evidence of interpulse mixing in the envelopes of ThMP-ing stars so that spectroscopic forensics to identify such a phase appears implausible. Nonetheless, from the point of view of understanding stars, the ThMPs constitute a beneficial computational laboratory to study fundamental aspects of secular stability of complex stellar models in advanced evolutionary stages pertinent also to the nature of thermally pulsing upper-AGB stars.

Acknowledgements. This study was initiated by H. Harzenmoser's inquisitive studying and digesting of the (also older) stellar-physical literature; A.G. is grateful for his persevering stimulus and for culinary support over the past years. This research has made use of NASA's Astrophysical Data System Abstract Service. The authors are most grateful for M. Gabriel's thorough refereeing efforts which led to significant improvements and eventually to a more coherent presentation of the material.

\section{Appendix A: Approximate stability analysis of stars in thermal imbalance}

In the following, the linear equations are compiled as they were used to compute the radial secular modes of stars in thermal imbalance. We relied on the perturbed Lagrangian quantities

$\zeta=\frac{\delta r}{r}, \quad p=\frac{\delta P}{P}, \quad t=\frac{\delta T}{T}, \quad l=\frac{\delta L}{x^{2} L_{*}}$.

The time dependence of the problem is factorized by $\exp (\mathrm{i} \sigma t)$. The eigenvalue of the resulting linear problem, $\sigma$, is normalized by $\|\sigma\|=\sqrt{3 G M_{*} / R_{*}^{3}}$. The linearized stellar structure equations for radiative regions, including approximated thermal-imbalance terms, are cast into the form

$x \mathrm{~d}_{x}\left(\begin{array}{c}\zeta \\ l \\ p \\ t\end{array}\right)=\left(\begin{array}{cccc}-3 & 0 & -\alpha & \delta \\ 0 & -2 & s_{23} & s_{24} \\ s_{31} & 0 & V & 0 \\ s_{41} & s_{42} & s_{43} & s_{44}\end{array}\right) \cdot\left(\begin{array}{c}\zeta \\ l \\ p \\ t\end{array}\right)$

with the secondary quantities in the matrix defined as

$$
\begin{aligned}
& s_{23}=C_{1}\left(C_{2} \varepsilon_{P}+T_{P}+\mathrm{i} \sigma \nabla_{\mathrm{ad}}\right), \\
& s_{24}=C_{1}\left(C_{2} \varepsilon_{T}+T_{T}-\mathrm{i} \sigma\right), \\
& s_{31}=\left(4+3 c_{1} \sigma^{2}\right) V, \\
& s_{41}=4 V \nabla_{\mathrm{rad}}, \\
& s_{42}=-V \nabla_{\mathrm{rad}} x^{2}\left(L_{*} / L_{r}\right), \\
& s_{43}=-V \nabla_{\mathrm{rad}} \varkappa_{P}, \\
& s_{44}=V \nabla_{\mathrm{rad}}\left(4-\varkappa_{T}\right),
\end{aligned}
$$

with $c_{1}=\left(r / R_{*}\right)^{3} /\left(M_{r} / M_{*}\right)$ and $x=r / R_{*}$. Furthermore,

$$
\begin{aligned}
& C_{1}=\frac{M_{r} U c_{P} T\|\sigma\|}{L_{*} x^{2}} \\
& C_{2}=\frac{\varepsilon}{c_{P} T\|\sigma\|} \\
& C_{3}=\frac{\mathrm{d}_{t} \log T}{\|\sigma\|} \\
& C_{4}=\nabla_{\mathrm{ad}} \frac{\mathrm{d}_{t} \log P}{\|\sigma\|}
\end{aligned}
$$

The quantities $U$ and $V$ represent the two homology invariants. The temporal derivatives of $\log P$ and $\log T$ are those used in the gravitational-energy term of the stellar-evolution computations. Finally, we approximate the thermal-imbalance terms as

$T_{P}=-C_{3} c_{P P}+C_{4}\left(\delta_{P}-\alpha\right)$
$T_{T}=-C_{3} c_{P T}+C_{4}\left(\delta_{T}+\delta\right)$

With $c_{P q}$ we refer to $\partial \log c_{P} / \partial \log q$, the same applies to $\delta_{q}$. The remaining, not explicitly defined variables have either their canonical meaning as used in stellar structure theory (see e.g. Kippenhahn \& Weigert 1994) or as referred to in Gautschy \& Glatzel (1990). Regularity constraints in the star's center enforce the central boundary conditions; at the stellar surface, the physically motivated ones used in Gautschy \& Glatzel (1990) were implemented.

\section{References}

Alexander, D., \& Ferguson, J. 1994, ApJ, 437, 879

Althaus, L., Serenelli, A., Córsico, A., \& Benvenuto, O. 2002, MNRAS, 330, 685 
Althaus, L. G., Serenelli, A. M., Córsico, A. H., \& Montgomery, M. H. 2003, A\&A, 404, 593

Bildsten, L. 1995, ApJ, 438, 852

Buchler, J., \& Perdang, J. 1979, ApJ, 231, 524

Cassisi, S., Iben, I., \& Tornambè, A. 1998, ApJ, 496, 376

Cox, J. 1980, Theory of Stellar Pulsation (Princeton, New Jersey: Princeton University Press)

Defouw, R. 1973, ApJ, 182, 215

Dennis, T. 1971, ApJ, 167, 311

Despain, K. 1981, ApJ, 251, 639

Eisenman, I. 2005, J. Phys. Oceanogr., 35, 616

Gabriel, M. 1972, A\&A, 18, 242

Gabriel, M., \& Noels, A. 1972, A\&A, 20, 455

Gautschy, A., \& Glatzel, W. 1990, MNRAS, 245, 154

Giannone, P., \& Weigert, A. 1967, ZfA, 67, 41

Glatzel, W. 1994, MNRAS, 271, 66

Gurevich, L., \& Lebedinski, A. 1947, JETP, 17, 792

Hansen, C. 1972, A\&A, 19, 71

Hansen, C. 1978, ARA\&A, 16, 15
Hansen, C., \& Van Horn, H. 1975, ApJ, 195, 735

Härm, R., \& Schwarzschild, M. 1972, ApJ, 172, 403

Iglesias, C., \& Rogers, F. 1996, ApJ, 464, 943

Kippenhahn, R., \& Weigert, A. 1994, Stellar Structure and Evolution (Berlin, Heidelberg: Springer Verlag)

Ledoux, P. 1962, in Star Evolution, Proceedings of the International School of Physics "Enrico Fermi", Course 28, ed. R. Gratton (New York: Academic Press), 394

Mazzitelli, I., \& D’Antona, F. 1986, ApJ, 308, 706

Paczyński, B. 1975, ApJ, 202, 558

Rose, W. 1966, ApJ, 146, 838

Schwarzschild, M., \& Härm, R. 1965, ApJ, 142, 855

Thomas, H.-C. 1967, ZfA, 67, 420

Trefethen, L. N., \& Embree, M. 2005, Spectra and Pseudospectra (Princeton University Press)

Weigert, A. 1965, Mitt. Astron. Gesell., 19, 61

Weigert, A. 1966, ZfA, 64, 395

Yoon, S.-C., Langer, N., \& van der Sluys, M. 2004, A\&A, 425, 207 